\title{
Computation of State Reachable Points of Descriptor Systems
}

\author{
Subashish Datta ${ }^{\dagger \S}$ and Volker Mehrmann ${ }^{\dagger}$
}

\begin{abstract}
This paper considers the problem of computing the state reachable points, from the origin, of a linear constant coefficient descriptor system. A numerical algorithm is proposed that can be implemented to characterize the reachable set in a numerically stable way. The original descriptor system is transformed into a strangeness-free system within the behavioral framework followed by a projection that separates the system into its differential and algebraic parts. It is shown that the computation of the image space of two matrices, associated with the projected system, is enough to compute the reachable set (from the origin). Moreover, a characterization is presented of all the inputs by which one can reach to any arbitrary points in the reachable set. The effectiveness of the proposed approach is demonstrated through numerical examples.

Index Terms - Linear descriptor system, behavior formulation, strangeness-free formulation, reachability
\end{abstract}

\section{INTRODUCTION}

The dynamical behavior of many practical systems can be modeled via descriptor systems (DS) where the system equation is a differential-algebraic equation, i.e. consists of a mixture of differential and algebraic equations. In the linear time-invariant case such systems have the general form

$$
E \dot{x}(t)=A x(t)+B u(t),
$$

where, if algebraic constraints are present, $E \in \mathbb{R}^{n \times n}$ is singular, $A \in \mathbb{R}^{n \times n}$ and $B \in \mathbb{R}^{n \times m}, x(t)$ is a state vector and $u(t)$ is input (control) to the system. Typically, also an initial condition $x\left(t_{0}\right)=x_{0}$ is considered. Examples are e.g. power system models, where the differential equations describe the dynamic behavior of electrical machines and other energy storage components while the algebraic constraints represent the power flow in the network [1]. Similarly, in spacecraft and robot trajectory planning, in addition to the differential equations, an algebraic relation defines a specific trajectory [2]. When modeling constrained mechanical systems like cranes and earth moving vehicles, a servo-constraint is described by an algebraic relation [3]. For further examples, see [4], [5], [6]. Descriptor systems are different from ordinary state-space systems in the sense that the system integrates and differentiates. The number of differentiations that are need is usually described by an index. There are many index concepts, see [7] for a comparison. In all cases (except for different counting) the index characterizes the smoothness requirements of the inhomogeneity, i.e., also that of the

This work was supported by DFG Collaborative Research Centre 910, Control of self-organizing nonlinear systems: Theoretical methods and concepts of application.

†Institut für Mathematik, MA 4-5, TU Berlin, Straße des 17. Juni 136, D-10623 Berlin, Germany; subashish@iitb.ac.in, mehrmann@math.tu-berlin.de

$\S$ This author was supported by Einstein Foundation Berlin, IMU Berlin Einstein Foundation Program. control function. If the inputs are piecewise continuous, then due to the differentiation, impulsive response may arise [6]. Hence, from the control theory perspective, an important question is to determine for a given system a smooth control input which transfers the DS from one state (say the origin) to another state in finite time. A related question is to compute the set of all state reachable points (via some chosen control inputs) of a DS. In this paper we present a systematic approach to address both these questions in a numerically computable way.

One of the traditional ways to carry out the analysis of DS is to transforming the system into an ordinary state space system governed by an ordinary differential equation (ODE), by resolving all the algebraic equations [4]. For instance, in a linearized power system, an ODE model is obtained from a DS by first expressing the algebraic variables in terms of the remaining variables and then eliminating them from the system (see e.g. the procedure in [1], [8]). However, in this way the algebraic constraints are not available any more and the system may drift off from the constraints, without being noticed. Also this procedure is numerically not advisable, several drawbacks are discussed in [4], [6].

Another approach for the analysis of DS, presented e.g. in [5], [9], is to decouple the original system into the fast (algebraic) and slow (differential) subsystem. To accomplish this, the coefficient matrices of the original system are transformed to Weierstrass canonical form. However, it has been observed that often ill-conditioned transformation matrices may arise in the computation of the Weierstrass canonical form [10], [11]. As a result, slight perturbations in the original DS may lead to largely perturbed subsystems (slow and fast). An alternative to the computation of the Weierstrass canonical form is to use so-called Wong sequences, see e.g. the recent work [12], but also this approach is not well suited for a numerical implementation. In [10] a staircase form based on orthogonal transformations is derived and implemented as a numerical method. This method is based on orthogonal transformations and can be implemented as a backward stable numerical methods.

All these techniques, however, rely on sequences of rank decisions which are difficult in finite precision arithmetic to compute some condensed or canonical form. There are wellknown techniques how to do this in the best possible way [13], however, in the context of the numerical integration of differential-algebraic equation another approach has been shown to be more robust. This alternative approach makes use of the fact that the system can be regularized by adding a sufficient number of derivatives of the original DS to the system, so that it becomes overdetermined but all necessary derivative information is available. The build-up 
of the derivative array is followed by the computation of two orthogonal projections to identify the differential and the algebraic equations. This derivative array approach [4], [6] has the advantage that is very robust to perturbations, and it has been implemented successfully in numerical simulation codes for linear and nonlinear descriptor systems [6]. Another surplus in this approach is that no changes of basis are carried out and thus the physical meaning of all the variables is preserved in the equivalent system. This is important in particular in the control context, when due to the application the inputs $u$ are clearly specified. In addition, since we use orthogonal projection matrices, the procedure can be implemented as a numerically stable algorithm.

In this paper we employ this approach for the computation of the state reachable points of a DS. For this, we first express the original DS in a behavior framework (making no distinction between the variables like states and inputs) [14]. Then, we construct an equivalent system (with the same solution set), called strangeness-free behavior system, by using derivative array consisting of the original DS and its derivatives. The minimum number of derivatives required to obtain a strangeness-free behavior model is referred to as strangeness-index of the behavior system [6]. From this derivative array then, via orthogonal transformations from the left, we filter two separate sets consisting of the differential and the algebraic equations, respectively. This new system is then strangeness-free in the behavior sense but not as a free system (with $u=0$ ). We could then employ the regularization method in [4] and rename variables to obtain a system that is strangeness-free as a free system, or we employ a preliminary feedback to achieve this. We follow this latter approach. Finally, to identify the reachable set, we use a recently developed projection representation [15] to obtain the solution as the sum of the solution of a purely differential and a purely algebraic system. In this way we obtain a new methodology to compute the state reachable points (from the origin) of a DS, and to characterize the reachable set.

A pictorial diagram to obtain a strangeness-free system is shown in Figure 1. To demonstrate the procedure we present the following example.

Example 1: Consider the DS $E \dot{x}=A x+B u$ with

$$
\left[\begin{array}{ll}
0 & 1 \\
0 & 0
\end{array}\right]\left[\begin{array}{l}
\dot{x}_{1} \\
\dot{x}_{2}
\end{array}\right]=\left[\begin{array}{cc}
1 & -1 \\
0 & 1
\end{array}\right]\left[\begin{array}{l}
x_{1} \\
x_{2}
\end{array}\right]+\left[\begin{array}{l}
0 \\
1
\end{array}\right] u
$$

Solving the second equation yields $x_{2}=-u$ and inserting it into the first equation yields $x_{1}=x_{2}+\dot{x}_{2}=-u-\dot{u}$ and hence if the input is e.g. a Heaviside function, then the solution contains an impulse. However, via an appropriate state feedback this behavior can be changed. Setting $u(t)=$ $u_{f}(t)+\tilde{u}(t)$, with a feedback $u_{f}=F x$, where $F=[1-1]$, we can obtain the closed loop system $E \dot{x}=(A+B F) x+B \tilde{u}$ given by $\left[\begin{array}{ll}0 & 1 \\ 0 & 0\end{array}\right] \dot{x}=\left[\begin{array}{cc}1 & -1 \\ 1 & 0\end{array}\right] x+\left[\begin{array}{l}0 \\ 1\end{array}\right] \tilde{u}$. Here we obtain $x_{1}=-\tilde{u}$ and inserting this, we have the standard state space system $\dot{x}_{2}=-x_{2}-\tilde{u}$, which does not contain any impulsive behavior.

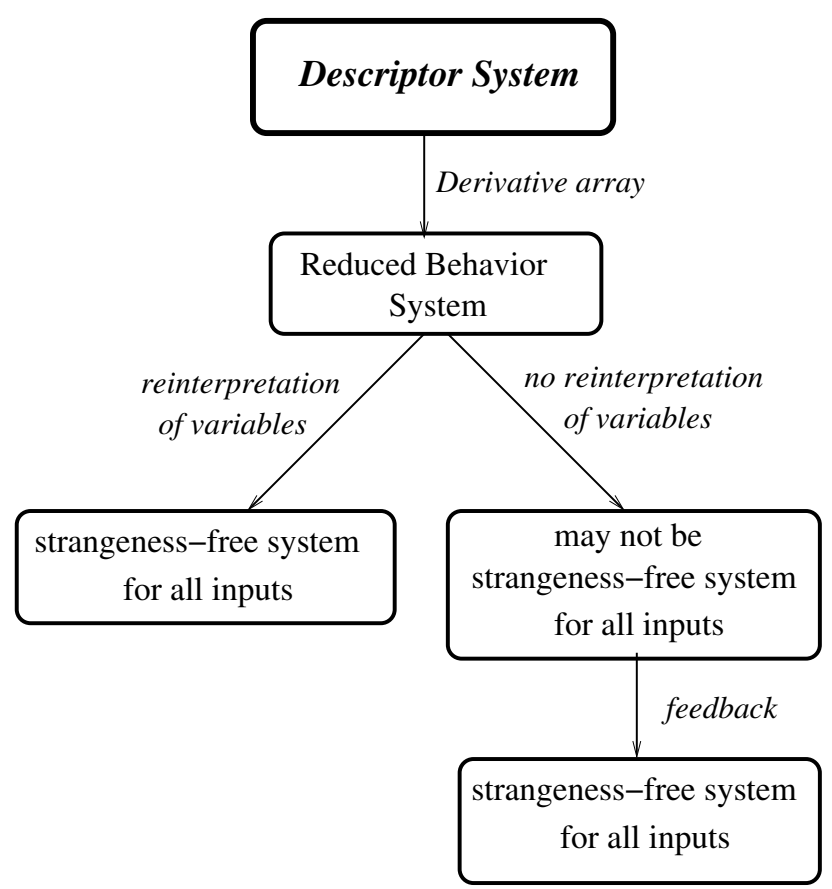

Fig. 1. Pictorial diagram to obtain a strangeness-free model from the given descriptor system.

The remainder of the paper is organized as follows. In Section II we briefly discuss the derivative array approach to obtain a strangeness-free behavior model associated with the original DS. The coefficient matrices associated with the transformed system are used in Section II-A to define two projection matrices. Then, we use the projection matrices to separate the DS into its differential and algebraic parts. We discuss the computation of the state reachable points of the original DS in Section III. A circuit example is presented in Section IV followed by concluding remarks in Section V.

\section{StRAngeness-FreE Formulation OF DS}

In this section we derive a strangeness-free behavior model associated with (1) via derivative array approach. For this we rewrite (1) as

$$
\mathbf{E} \dot{z}(t)=\mathbf{A} z(t),
$$

where

$$
\mathbf{E}:=\left[\begin{array}{ll}
E & \mathbf{0}
\end{array}\right] \in \mathbb{R}^{n \times(n+m)}, \mathbf{A}:=[A B] \in \mathbb{R}^{n \times(n+m)},
$$

by introducing a new variable $z=\left[\begin{array}{ll}x^{T} & u^{T}\end{array}\right]^{T}$. For the time being let us formally assume that $x(t)$ and $u(t)$ are at least $\mu>0$ times differentiable. We will drop this assumption later, once the projection matrices $Z_{1}, Z_{2}$ and $T_{2}$ are computed from the derivative array. Subsequently, we will use only these three matrices to obtain a strangeness-free system of (2).

By performing a sequence of differentiations of (2) and stacking all together, we get

$$
\mathcal{M}_{\mu} \dot{z}_{\mu}=\mathcal{N}_{\mu} z_{\mu},
$$


$\mathcal{M}_{\mu}=\left[\begin{array}{ccccc}\mathbf{E} & \mathbf{0} & \cdots & \mathbf{0} & \mathbf{0} \\ -\mathbf{A} & \mathbf{E} & \cdots & \mathbf{0} & \mathbf{0} \\ \vdots & \vdots & \ddots & \vdots & \vdots \\ \mathbf{0} & \mathbf{0} & \cdots & -\mathbf{A} & \mathbf{E}\end{array}\right] ; \mathcal{N}_{\mu}=\left[\begin{array}{cccc}\mathbf{A} & \mathbf{0} & \cdots & \mathbf{0} \\ \mathbf{0} & \mathbf{0} & \cdots & \mathbf{0} \\ \vdots & \vdots & \ddots & \vdots \\ \mathbf{0} & \mathbf{0} & \cdots & \mathbf{0}\end{array}\right]$

and $z_{\mu}=\left[\begin{array}{llll}z^{T} & \dot{z}^{T} & \ldots & z^{(\mu)^{T}}\end{array}\right]^{T}$. Note that (4) stacks the original system (1) and its derivatives up to level $\mu$. The sequence of derivatives in (4) must be continued until the following hypothesis holds [6].

Hypothesis 1: There exist integers $\mu, d, a$ such that the inflated pair $\left(\mathcal{M}_{\mu}, \mathcal{N}_{\mu}\right)$ in (4) has the following properties:

1) $\operatorname{rank}\left(\mathcal{M}_{\mu}\right)=(\mu+1) n-a$. This implies the existence of a matrix $Z_{2} \in \mathbb{R}^{(\mu+1) n \times a}$ with orthonormal columns and maximal rank $a$, satisfying $Z_{2}^{T} \mathcal{M}_{\mu}=0$.

2) Denoting $\widehat{A}_{2}:=Z_{2}^{T} \mathcal{N}_{\mu}\left[\begin{array}{c}I_{n+m} \\ \mathbf{0}\end{array}\right] \in \mathbb{R}^{a \times(n+m)}$, we have $\operatorname{rank}\left(\widehat{A}_{2}\right)=a$, and there exists $T_{2} \in \mathbb{R}^{(n+m) \times d}$ having orthonormal columns satisfying $\widehat{A}_{2} T_{2}=0$.

3) $\operatorname{rank}\left(\mathbf{E} T_{2}\right)=d$. This implies the existence of a matrix $Z_{1} \in \mathbb{R}^{n \times d}$ with orthonormal columns satisfying maximal $\operatorname{rank}\left(\widehat{E}_{1}\right)=d$ where $\widehat{E}_{1}=Z_{1}^{T} \mathbf{E} \in \mathbb{R}^{d \times(n+m)}$.

We refer to the smallest $\mu \geq 0$ for which the above hypothesis holds as strangeness-index of (2). Once the orthonormal matrices $Z_{1}, Z_{2}$ and $T_{2}$ are constructed according to Hypothesis 1, we obtain a strangeness-free system of (2) according to the following result.

Theorem 1: [4], [6] Assume that Hypothesis 1 holds for an inflated pair $\left(\mathcal{M}_{\mu}, \mathcal{N}_{\mu}\right)$, corresponding to the pair $(\mathbf{E}, \mathbf{A})$. Consider the strangeness-free system

$$
\left[\begin{array}{c}
\widehat{E}_{1} \\
\mathbf{0}
\end{array}\right] \dot{z}=\left[\begin{array}{l}
\widehat{A}_{1} \\
\widehat{A}_{2}
\end{array}\right] z
$$

with coefficients $\widehat{E}_{1} \in \mathbb{R}^{d \times(n+m)}, \widehat{A}_{1} \in \mathbb{R}^{d \times(n+m)}, \widehat{A}_{2} \in$ $\mathbb{R}^{a \times(n+m)}$ given by

$\widehat{E}_{1}=Z_{1}^{T} \mathbf{E}, \widehat{A}_{1}=Z_{1}^{T} \mathbf{A}, \widehat{A}_{2}=Z_{2}^{T} \mathcal{N}_{\mu}\left[\begin{array}{c}I_{n+m} \\ \mathbf{0}\end{array}\right] \in \mathbb{R}^{a \times(n+m)}$

Then, the differential-algebraic system (2) and the strangeness-free system (5) have the same solution set.

Example 2: Consider the DS $E \dot{x}=A x+B u$ from Example 1, which is already strangeness-free in the behavior sense and thus with $Z_{2}=\left[\begin{array}{ll}0 & 1\end{array}\right]^{T}$ and $Z_{1}=\left[\begin{array}{ll}1 & 0\end{array}\right]^{T}$ the strangeness-free behavior system is obtained.

Once the strangeness-free behavior system is available, it is possible to check the consistency of initial conditions, i.e., whether $x\left(t_{0}\right)=x_{0}$ is consistent with the algebraic equation $\widehat{A}_{2} z_{0}=\mathbf{0}$ for all possible input functions $u$. If this is not the case, then the input function will be restricted in its initial value through the equation $\widehat{A}_{2} z\left(t_{0}\right)=\widehat{A}_{2}\left[\begin{array}{ll}x_{0}^{T} & u\left(t_{0}\right)^{T}\end{array}\right]^{T}=\mathbf{0}$.

Using (3), the strangeness-free system (5) can be represented as follows:

$$
\begin{aligned}
\widetilde{E}_{1} \dot{x}(t) & =\widetilde{A}_{1} x(t)+\widetilde{B}_{1} u(t) \\
\mathbf{0} & =\widetilde{A}_{2} x(t)+\widetilde{B}_{2} u(t)
\end{aligned}
$$

where $\widetilde{E}_{1}=Z_{1}^{T} E \in \mathbb{R}^{d \times n}$,

$$
\begin{aligned}
& \widetilde{A}_{1}=\widehat{A}_{1}\left[\begin{array}{c}
I_{n} \\
\mathbf{0}
\end{array}\right] \in \mathbb{R}^{d \times n}, \quad \widetilde{A}_{2}=\widehat{A}_{2}\left[\begin{array}{c}
I_{n} \\
\mathbf{0}
\end{array}\right] \in \mathbb{R}^{a \times n} \\
& \widetilde{B}_{1}=\widehat{A}_{1}\left[\begin{array}{c}
\mathbf{0} \\
I_{m}
\end{array}\right] \in \mathbb{R}^{d \times m}, \quad \widetilde{B}_{2}=\widehat{A}_{2}\left[\begin{array}{c}
\mathbf{0} \\
I_{m}
\end{array}\right] \in \mathbb{R}^{a \times m} .
\end{aligned}
$$

Since in the behavior setting, there is no distinction between the states and input variables, the resulting transformed system (6) may not be strangeness-free as a free system with $u=0$. If a reinterpretation of variables makes no sense from the point of view of the considered application, because $x(t)$ and $u(t)$ in (1) are specified as state and input, respectively, then the only way to achieve a strangeness-free system for all possible inputs, is to use a state feedback that achieves this property, see Example 1 and the detailed discussion about this topic and also on the choice of initial conditions in [4]. In the context of computing the set of reachable points (from the origin) of system (1) and if no reinterpretation of variables is possible, then an appropriate initial feedback is the only option.

Definition 1: A vector $x_{1} \in \mathbb{R}^{n}$ is said to be reachable from the initial condition $x\left(t_{0}\right)=x_{0}$, if there exists a sufficiently smooth control input $u(t)$ and a finite time $t_{1}>0$ such that $x\left(t_{1}\right)=x_{1}$.

Note that for reachability the initial condition has to be consistent and the set of initial values for the control may be restricted as well, because they two together have to satisfy the algebraic constraint.

In the following section we will use a projection method to determine the reachable set of system (1). More details on projector based analysis of the differential-algebraic systems can be found in [16].

\section{A. An Equivalent Projected System}

Let us rewrite (6) as

$$
\mathcal{E} \dot{x}=\mathcal{A} x+\mathcal{B} u
$$

where $\mathcal{E}=\left[\begin{array}{c}\widetilde{E}_{1} \\ \mathbf{0}\end{array}\right], \mathcal{A}=\left[\begin{array}{c}\widetilde{A}_{1} \\ \widetilde{A}_{2}\end{array}\right]$ and $\mathcal{B}=\left[\begin{array}{c}\widetilde{B}_{1} \\ \widetilde{B}_{2}\end{array}\right]$, and define the two subspaces: $\mathbb{E}_{d}:=\operatorname{Im}\left(\mathcal{E}^{T}\right), \mathbb{E}_{a}:=\operatorname{ker}(\mathcal{E})$ where Im and ker denote the image and kernel space, respectively. Note that $\mathbb{E}_{d}$ and $\mathbb{E}_{a}$ are orthogonal complements to each other. Corresponding to these two subspaces, we can now partition the state variable $x(t)$ additively into two parts $x_{d}(t)$ and $x_{a}(t)$, which we will call differential and algebraic components of $x(t)$ respectively. To obtain $x_{d}$ and $x_{a}$, let us define the two projectors

$$
P_{d}=\mathcal{E}^{+} \mathcal{E}, \quad \text { and } \quad P_{d}^{\prime}=I-\mathcal{E}^{+} \mathcal{E}
$$

where $\mathcal{E}^{+}$is the Moore-Penrose inverse of $\mathcal{E}$, [17]. Note that $P_{d}$ is an orthogonal projector onto the subspace $\mathbb{E}_{d}$, whereas $P_{a}$ is an orthogonal projector onto the subspace $\mathbb{E}_{a}$, and we we set

$$
x_{d}(t):=P_{d} x(t), x_{a}(t)=P_{d}^{\prime} x(t) .
$$


Then, according to definition (8), we have $x(t)=x_{d}(t)+$ $x_{a}(t)$. Additionally, let us define another projector $Q$ onto $\operatorname{Im}(\mathcal{E})$ via

$$
Q:=\mathcal{E E}^{+}=\left[\begin{array}{cc}
I_{d} & \mathbf{0} \\
\mathbf{0} & \mathbf{0}
\end{array}\right] \quad \text { and } \quad Q^{\prime}=I-\mathcal{E \mathcal { E }}^{+} .
$$

Then, we have the following result.

Theorem 2: [15, Theorem 4.4] Let the projectors $P_{d}$ and $P_{d}^{\prime}$ be as in (7) and the variables $x_{d}$ and $x_{a}$ as in (8). Then, $x(t)$ is a solution of (1) if and only if $x_{d}(t)$ and $x_{a}(t)$ are solutions of the system

$$
\begin{aligned}
& \dot{x}_{d}(t)=G_{d} x_{d}(t)+B_{d} u(t) \\
& x_{a}(t)=G_{a} x_{d}(t)+B_{a} u(t)
\end{aligned}
$$

where

$$
\begin{aligned}
G_{a} & :=-\left(Q^{\prime} \mathcal{A} P_{d}^{\prime}\right)^{+}\left(Q^{\prime} \mathcal{A} P_{d}\right), \quad G_{d}:=\mathcal{E}^{+} \mathcal{A}\left(P_{d}+G_{a}\right), \\
B_{a} & :=-\left(Q^{\prime} \mathcal{A} P_{d}^{\prime}\right)^{+} \mathcal{B}, \quad B_{d}:=\mathcal{E}^{+} \mathcal{B}+\mathcal{E}^{+} \mathcal{A} B_{a}
\end{aligned}
$$

Moreover, an initial value $x_{0}$ is consistent if and only if it satisfies the following relation at initial time $t_{0}$ : $\left(P_{d}^{\prime}-\right.$ $\left.G_{a}\right) x_{0}=B_{a} u\left(t_{0}\right)$.

Theorem 2 states that a solution $x(t)$ of (1) can be computed by solving system (10) and forming $x(t)=$ $x_{d}(t)+x_{a}(t)$. Hence, the set of reachable points of system (1) can be determined by computing the state reachable points of system (10). Thus, we can rephrase the Definition of reachability as follows:

A vector $x_{1}:=x_{d_{1}}+x_{a_{1}}$ is said to be reachable from a consistent initial condition $x\left(t_{0}\right)=x_{0}$, if there exists a sufficiently smooth control input $u(t)$ and a finite time $t_{1}>0$ such that $x_{d}\left(t_{1}\right)=x_{d_{1}}$ and $x_{a}\left(t_{1}\right)=x_{a_{1}}$.

In the following section we compute the set of state reachable points from the consistent initial condition $x\left(t_{0}\right)=$ $x_{0}$ of system (1) by using the solution set of system (10).

\section{Computing the Reachable Set}

Following the discussion in Section II it is clear that a reachable point $x_{1}$ of (1) can be determined by computing its differential complement $x_{d_{1}}$ and algebraic component $x_{a_{1}}$ via the relations given in (10). In this section, we first compute the differential component $x_{d_{1}}$ of a reachable point $x_{1}$ from the relation $\dot{x}_{d}(t)=G_{d} x_{d}(t)+B_{d} u(t)$. Then, we use $x_{a}(t)=G_{a} x_{d}(t)+B_{a} u(t)$ to compute the algebraic component $x_{a_{1}}$ of $x_{1}$.

Let us assume that the initial state $x_{0}$ of (1) is consistent. Then, the differential component $x_{d_{0}}$ of $x_{0}$ would be $x_{d_{0}}=$ $P_{d} x_{0}$ which is an initial condition for the standard state space system $\dot{x}_{d}=G_{d} x_{d}+B_{d} u$. The differential component of a state trajectory $x(t)$, i.e. the solution of the differential equation in (10) then has the form

$$
x_{d}(t)=e^{G_{d}\left(t-t_{0}\right)} x_{d_{0}}+\int_{t_{0}}^{t} e^{G_{d}(t-\tau)} B_{d} u(\tau) d \tau
$$

and the resulting algebraic component of $x(t)$ would be

$$
\begin{aligned}
x_{a}(t) & =G_{a}\left[e^{G_{d}\left(t-t_{0}\right)} x_{d_{0}}+\int_{t_{0}}^{t} e^{G_{d}(t-\tau)} B_{d} u(\tau) d \tau\right] \\
& +B_{a} u(t) .
\end{aligned}
$$

Then it is clear that the state responses $x_{d}(t)$ and $x_{a}(t)$ are uniquely determined by the initial condition $x_{0}$, the control input $u(\tau)$ for $t_{0} \leq \tau \leq t$ and the initial time $t_{0}$. Assuming initial time $t_{0}=0$ and initial condition $x_{0}=0$, then we have $x_{d_{0}}=P_{d} x_{0}=0$ and hence the solution (12) is given by

$$
x_{d}(t)=\int_{0}^{t} e^{G_{d}(t-\tau)} B_{d} u(\tau) d \tau .
$$

Let us define an operator $\mathcal{L}_{d}(u, t)$ via

$$
\mathcal{L}_{d}(u, t):=\int_{0}^{t} e^{G_{d}(t-\tau)} B_{d} u(\tau) d \tau .
$$

Then, the set of reachable points $x_{d_{1}}$ from the origin is essentially the set of points which are in the image space of $\mathcal{L}_{d}(u, t)$ (denoted as $\operatorname{Im}\left[\mathcal{L}_{d}(u, t)\right]$ ). In the following result we show that $\operatorname{Im}\left[\mathcal{L}_{d}(u, t)\right]$ is equivalent to the image space of the symmetric matrix $W(p, t)$ given by

$$
W(p, t):=\int_{0}^{t} p(\tau) e^{G_{d}(t-\tau)} B_{d} B_{d}^{T} e^{G_{d}^{T}(t-\tau)} p(\tau) d \tau
$$

where $p(\tau)$ is a polynomial which is not identically zero.

Lemma 1: Let $W(p, t)$ be as in (15). Then, we have

$$
\operatorname{Im}[W(p, t)]=\operatorname{Im}\left[\mathcal{L}_{d}(u, t)\right] .
$$

Proof: First, we show that $\operatorname{Im}[W(p, t)] \subseteq \operatorname{Im}\left[\mathcal{L}_{d}(u, t)\right]$. Let us consider a vector $x_{d_{1}}$ such that $x_{d_{1}} \in \operatorname{Im}[W(p, t)]$. Then, there exists a vector $w \in \mathbb{R}^{n}$ such that $W(p, t) w=$ $x_{d_{1}}$. Now, by choosing the input

$$
u(\tau)=p^{2}(\tau) B_{d}^{T} e^{G_{d}^{T}(t-\tau)} w, 0 \leq \tau \leq t
$$

we have

$$
\begin{aligned}
\mathcal{L}_{d}(u, t) & =\left[\int_{0}^{t} p(\tau) e^{G_{d}(t-\tau)} B_{d} B_{d}^{T} e^{G_{d}^{T}(t-\tau)} p(\tau) d \tau\right] w \\
& =W(p, t) w=x_{d_{1}},
\end{aligned}
$$

and hence, $x_{d_{1}} \in \operatorname{Im}\left[\mathcal{L}_{d}(u, t)\right]$. Since $x_{d_{1}}$ is chosen arbitrarily we have $\operatorname{Im}[W(p, t)] \subseteq \operatorname{Im}\left[\mathcal{L}_{d}(u, t)\right]$.

To show the converse implication $\operatorname{Im}\left[\mathcal{L}_{d}(u, t)\right] \subseteq$ $\operatorname{Im}[W(p, t)]$, let $x_{d_{1}} \in \operatorname{Im}\left[\mathcal{L}_{d}(u, t)\right]$. Then there exists an input $u_{1}(t)$ such that $\mathcal{L}_{d}\left(u_{1}, t\right)=x_{d_{1}}$. Suppose that $x_{d_{1}} \notin$ $\operatorname{Im}[W(p, t)]$, then $\operatorname{ker}[W(p, t)]$ is nonempty and we can find a vector $k \in \operatorname{ker}[W(p, t)]$ such that $k^{T} x_{d_{1}} \neq 0$, i.e. $k^{T} W(p, t) k=0$. This implies that

$$
\int_{0}^{t}\left[k^{T} p(\tau) e^{G_{d}(t-\tau)} B_{d}\right]\left[k^{T} p(\tau) e^{G_{d}(t-\tau)} B_{d}\right]^{T} d \tau=0
$$

and therefore $\int_{0}^{t}\left\|k^{T} p(\tau) e^{G_{d}(t-\tau)} B_{d}\right\|_{2}^{2} d \tau=0$, which means that $k^{T} p(\tau) e^{G_{d}(t-\tau)} B_{d}=0$ for all $\tau \in[0, t]$. Since $p(\tau)$ is not identically zero, we obtain

$$
k^{T} e^{G_{d}(t-\tau)} B_{d}=0, \quad \forall \tau \in[0, t],
$$

which implies that $k^{T} x_{d_{1}}=k^{T} \mathcal{L}_{d}\left(u_{1}, t\right)=$ $\int_{0}^{t} k^{T} e^{G_{d}(t-\tau)} B_{d} u_{1} d \tau=0$. However, we had chosen $k^{T}$ such that $k^{T} x_{d_{1}} \neq 0$ and hence we have reached at a contradiction. Hence $\operatorname{Im}\left[\mathcal{L}_{d}(u, t)\right] \subseteq \operatorname{Im}[W(p, t)]$ and the proof is complete. 
According to Lemma 1 the set of all reachable points $x_{d_{1}}$ (from the origin) due to the state response $x_{d}(t)$ is essentially the set of points which belong to $\operatorname{Im}[W(p, t)]$. Moreover, by defining the subspace

$$
\mathcal{X}_{d}:=\operatorname{Im}\left(\mathcal{C}_{d}\right),
$$

where $\mathcal{C}_{d}=\left[\begin{array}{llll}B_{d} & G_{d} B_{d} & \cdots & G_{d}^{n-1} B_{d}\end{array}\right]$ it follows from the proof in [5, Lemma 2.1.1] that

$$
\operatorname{Im}[W(p, t)]=\mathcal{X}_{d} .
$$

So far we have characterized the reachability set for the dynamical part, but we also need to discuss the restrictions that are forced by the algebraic part. Recall that the algebraic component of a state $x$ is determined by $x_{a}(t)=G_{a} x_{d}(t)+$ $B_{a} u(t)$. Hence, to compute the algebraic component $x_{a_{1}}$ of a reachable point $x_{1}$, assume that $x_{d_{1}}$ is a reachable point due to the state response $x_{d}(t)$ with some input $u_{1}(t)$ and a finite time $t_{1}>0$. Then, corresponding to $u_{1}(t)$ and $t_{1}$, the algebraic component $x_{a_{1}}$ is given by: $x_{a_{1}}=G_{a} x_{d_{1}}+$ $B_{a} u_{1}\left(t_{1}\right)$. Since $x_{d_{1}} \in \mathcal{X}_{d}$, we can write $x_{d_{1}}=\mathcal{C}_{d} y$ for some $y \in \mathbb{R}^{n m}$. Hence, $x_{a_{1}}$ takes the form

$$
x_{a_{1}}=G_{a} \mathcal{C}_{d} y+B_{a} c,
$$

where $c=u_{1}\left(t_{1}\right) \in \mathbb{R}^{m}$. Hence, the algebraic component of a reachable point $x_{1}$ is determined by the relation (19). Defining the set

$$
\mathcal{X}_{a}:=\left\{x_{a_{1}} \in \mathbb{R}^{n} \mid x_{a_{1}}=G_{a} \mathcal{C}_{d} y+B_{a} c, c=u\left(t_{1}\right)\right\},
$$

where $y \in \mathbb{R}^{m n}$ and $u(t)$ is an input to system (10), then, $\mathcal{X}_{a}$ consists of all the algebraic components of the reachable points $x_{1}$. Note that $\mathcal{X}_{a}=\operatorname{Im}\left[G_{a} \mathcal{C}_{d} B_{a}\right]$ and thus we have the complete characterization of the set $\mathcal{R}_{0}$ of reachable points of system (1) from an initial condition $x_{0}=0$ at $t_{0}=0$.

Theorem 3: Let $\mathcal{X}_{d}$ and $\mathcal{X}_{a}$ be as in (17) and (20) respectively. Then,

$$
\mathcal{R}_{0}=\mathcal{X}_{d}+\mathcal{X}_{a}
$$

where $\mathcal{X}_{d}+\mathcal{X}_{a}:=\left\{x_{d_{1}}+x_{a_{1}} \mid x_{d_{1}} \in \mathcal{X}_{d}, x_{a_{1}} \in \mathcal{X}_{a}\right\}$.

Proof: We will show first, that $\mathcal{R}_{0} \subseteq \mathcal{X}_{d}+\mathcal{X}_{a}$. If $x_{1} \in$ $\mathcal{R}_{0}$, then there exists an input $u(t)$ and a finite time $t_{1}$ such that $x_{d}\left(t_{1}\right)=x_{d_{1}}$ and $x_{a}\left(t_{1}\right)=x_{a_{1}}$. In addition to this, $x_{1}=x_{d_{1}}+x_{a_{1}}$. Hence, we only have to show that $x_{d_{1}} \in \mathcal{X}_{d}$ and $x_{a_{1}} \in \mathcal{X}_{a}$.

Recall that $x_{d}(t)=\int_{0}^{t} e^{G_{d}(t-\tau)} B_{d} u(\tau) d \tau$. It is well known, see [5], that corresponding to a matrix $G_{d} \in \mathbb{R}^{n \times n}$ there exists continuous functions $\alpha_{i}(t)$ for $i=1,2, \cdots, n-1$ such that

$$
e^{G_{d} t}=\alpha_{0}(t) I+\alpha_{1}(t) G_{d}+\cdots+\alpha_{n-1}(t) G_{d}^{n-1} .
$$

Then, using (21), we obtain

$$
\begin{array}{r}
x_{d}(t)=\int_{0}^{t} \alpha_{0}(t-\tau) B_{d} u(\tau) d \tau+\int_{0}^{t} \alpha_{1}(t-\tau) G_{d} B_{d} u(\tau) d \tau+ \\
\cdots+\int_{0}^{t} \alpha_{n-1}(t-\tau) G_{d}^{n-1} B_{d} u(\tau) d \tau \\
=\left[\begin{array}{llll}
B_{d} & G_{d} B_{d} & \cdots & G_{d}^{n-1} B_{d}
\end{array}\right]\left[\begin{array}{c}
\int_{0}^{t} \alpha_{0}(t-\tau) u(\tau) d \tau \\
\int_{0}^{t} \alpha_{1}(t-\tau) u(\tau) d \tau \\
\vdots \\
\int_{0}^{t} \alpha_{n-1}(t-\tau) u(\tau) d \tau
\end{array}\right] .
\end{array}
$$

Hence $x_{d_{1}} \in \mathcal{X}_{d}$. Since $x_{d_{0}}=0$, the algebraic component $x_{a}(t)$ in (13) has the form

$$
x_{a}(t)=G_{a}\left[\int_{0}^{t} e^{G_{d}(t-\tau)} B_{d} u(\tau) d \tau\right]+B_{a} u(t),
$$

and

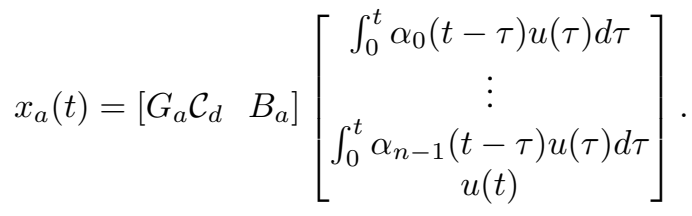

Therefore, $x_{a_{1}} \in \mathcal{X}_{a}$, which proves that $\mathcal{R}_{0} \subseteq \mathcal{X}_{d}+\mathcal{X}_{a}$. To show that $\mathcal{X}_{d}+\mathcal{X}_{a} \subseteq \mathcal{R}_{0}$, let us consider a vector $x_{1} \in \mathcal{X}_{d}+$ $\mathcal{X}_{a}$. Then, according to the definition of $\mathcal{X}_{d}+\mathcal{X}_{a}$, we can find $x_{d_{1}} \in \mathcal{X}_{d}$ and $x_{a_{1}} \in \mathcal{X}_{a}$ such that $x_{1}=x_{d_{1}}+x_{a_{1}}$. Hence, we only have to show that there exists an input $u(t)$ and finite time $t_{1}>0$ such that $x_{d}\left(t_{1}\right)=x_{d_{1}}$ and $x_{a}\left(t_{1}\right)=x_{a_{1}}$.

Since $x_{d_{1}} \in \mathcal{X}_{d}$, it follows form (18) that $x_{d_{1}} \in$ $\operatorname{Im}[W(p, t)]$. Hence, we can find a vector $w \in \mathbb{R}^{n}$ such that $x_{d_{1}}=W(p, t) w$. Let us choose a polynomial $p(\tau)=\tau^{\mu}$ for any fixed $\tau>0$. Then, corresponding to the chosen $w$ to obtain $x_{d_{1}}$, let us construct an input via

$$
u(\tau)=\tau^{2 \mu} B_{d}^{T} e^{G_{d}^{T}(t-\tau)} w, 0 \leq \tau \leq t .
$$

The resulting state response $x_{d}(t)$ then is

$$
\begin{aligned}
x_{d}(t) & =\int_{0}^{t} e^{G_{d}(t-\tau)} B_{d} u(\tau) d \tau \\
& =\left[\int_{0}^{t} \tau^{\mu} e^{G_{d}(t-\tau)} B_{d} B_{d}^{T} e^{G_{d}^{T}(t-\tau)} \tau^{\mu} d \tau\right] w .
\end{aligned}
$$

Since the polynomial $p(\tau)=\tau^{\mu}$ is not identically zero, the matrix $W(p, t)$ is well defined (see (15)) and it follows from (23) that $x_{d}(t)=W(p, t) w=x_{d_{1}}$.

Now, since $x_{a_{1}} \in \mathcal{X}_{a}$, there exist two vectors $y \in \mathbb{R}^{m n}$ and $c \in \mathbb{R}^{m}$ such that $x_{a_{1}}=G_{a} \mathcal{C}_{d} y+B_{a} c$. Corresponding to the input $u(\tau)$ defined in (22), the algebraic component of the state variable $x(t)$ then is

$$
\begin{aligned}
x_{a}(t) & =G_{a} \int_{0}^{t} e^{G_{d}(t-\tau)} B_{d} u(\tau) d \tau+B_{a} u(t) \\
& =G_{a}\left[\int_{0}^{t} \tau^{\mu} e^{G_{d}(t-\tau)} B_{d} B_{d}^{T} e^{G_{d}^{T}(t-\tau)} \tau^{\mu} d \tau\right] w+ \\
& t^{2 \mu} B_{a} B_{d}^{T} e^{G_{d}^{T}(t-t)} w \\
& =G_{a} W(p, t) w+t^{2 \mu} B_{a} B_{d}^{T} w \\
& =G_{a} x_{d_{1}}+t^{2 \mu} B_{a} B_{d}^{T} w
\end{aligned}
$$

Setting $c=t^{2 \mu} B_{d}^{T} w$, we have $x_{a}(t)=G_{a} x_{d_{1}}+B_{a} c$, hence we only have to show that there exists a vector $y$ such that $\mathcal{C}_{d} y=x_{d_{1}}$. Note that the vector $x_{d_{1}}$ is in the image space of $W(p, t)$. Since, $\operatorname{Im}\left(\mathcal{C}_{d}\right)=\operatorname{Im}[W(p, t)]$, we can find $y$ such that $\mathcal{C}_{d} y=x_{d_{1}}$. Hence $\mathcal{X}_{d}+\mathcal{X}_{a} \subseteq \mathcal{R}_{0}$ and the proof is complete.

Theorem 3 characterizes the reachable set of the DS (1) from the origin. In addition, the characterization of all the inputs $u(t)$ by which one can reach to any arbitrary points in the reachable set is given by (22). Since, $\mathcal{X}_{d}=\operatorname{Im}\left(\mathcal{C}_{d}\right)$ and 
$\mathcal{X}_{a}=\operatorname{Im}\left[G_{a} \mathcal{C}_{d} \quad B_{a}\right]$, the set $\mathcal{R}_{0}$ can directly be computed using the matrices defined in (11). Moreover, the dimension of the subspace $\mathcal{R}_{0}$ is $\operatorname{dim}\left(\mathcal{R}_{0}\right)=\operatorname{dim}\left(\mathcal{X}_{d}\right)+\operatorname{dim}\left(\mathcal{X}_{a}\right)$ where dim refers to the dimension of a subspace.

To demonstrate the proposed procedure, in the following section, we compute the reachable set of an electrical circuit.

\section{A Circuit ExAmple}

Let us consider an electrical circuit as shown in Fig. 2, see[5], [9]. Assuming that the voltages in capacitors $v_{c_{1}}, v_{c_{2}}$ and currents $i_{1}, i_{2}$ as states, the dynamic of the electrical circuit can be represented as $E \dot{x}(t)=A x(t)+B v_{s}(t)$, where $x(t)=\left[\begin{array}{llll}v_{c_{1}}(t) & v_{c_{2}}(t) & i_{2}(t) & i_{1}(t)\end{array}\right]^{T}, v_{s}(t)$ is the input voltage to the circuit and

$E=\left[\begin{array}{cccc}C_{1} & 0 & 0 & 0 \\ 0 & C_{2} & 0 & 0 \\ 0 & 0 & -L & 0 \\ 0 & 0 & 0 & 0\end{array}\right], A=\left[\begin{array}{cccc}0 & 0 & 0 & 1 \\ 0 & 0 & 1 & 0 \\ -1 & 1 & 0 & 0 \\ 1 & 0 & 0 & R\end{array}\right], B=\left[\begin{array}{c}0 \\ 0 \\ 0 \\ -1\end{array}\right]$

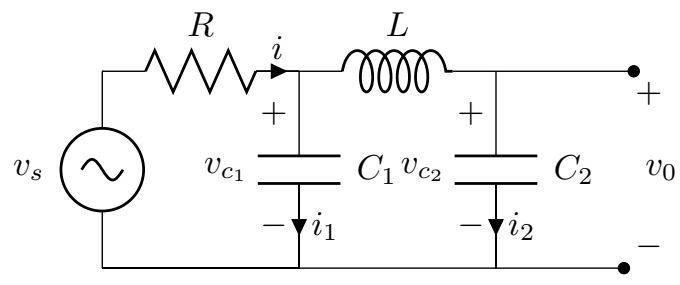

Fig. 2. An $R L C$ electrical circuit with input voltage $v_{s}$. The voltages across the capacitors $C_{1}$ and $C_{2}$ are $v_{c_{1}}$ and $v_{c_{2}}$ respectively. The loop currents are $i_{1}$ and $i_{2}$.

Let us assume that all the values of resistor, capacitors and inductor are one. Then, according to Hypothesis 1, it can be verified that the above system is strangeness-free in the behavior framework. Hence, we have the following matrices

$$
\begin{aligned}
& \widetilde{E}_{1}=\left[\begin{array}{cccc}
1 & 0 & 0 & 0 \\
0 & 1 & 0 & 0 \\
0 & 0 & -1 & 0
\end{array}\right], \widetilde{A}_{1}=\left[\begin{array}{cccc}
0 & 0 & 0 & 1 \\
0 & 0 & 1 & 0 \\
-1 & 1 & 0 & 0
\end{array}\right], \widetilde{B}_{1}=\left[\begin{array}{l}
0 \\
0 \\
0
\end{array}\right] \\
& \widetilde{A}_{2}=\left[\begin{array}{llll}
1 & 0 & 0 & 1
\end{array}\right], \widetilde{B}_{2}=-1 .
\end{aligned}
$$

Corresponding to theses matrices, (11) will take the following form:

$$
\begin{aligned}
G_{d} & =\left[\begin{array}{cccc}
-1 & 0 & 0 & 0 \\
0 & 0 & 1 & 0 \\
1 & -1 & 0 & 0 \\
0 & 0 & 0 & 0
\end{array}\right], G a=\left[\begin{array}{cccc}
0 & 0 & 0 & 0 \\
0 & 0 & 0 & 0 \\
0 & 0 & 0 & 0 \\
-1 & 0 & 0 & 0
\end{array}\right] \\
B_{d} & =\left[\begin{array}{llll}
1 & 0 & 0 & 0
\end{array}\right]^{T}, B_{a}=\left[\begin{array}{llll}
0 & 0 & 0 & 1
\end{array}\right]^{T} .
\end{aligned}
$$

Then, the reachable space from the origin, is given by $\mathcal{R}_{0}=$ $\mathcal{X}_{d}+\mathcal{X}_{a}$, where

$$
\mathcal{X}_{d}=\operatorname{Im}\left(\mathcal{C}_{d}\right)=\operatorname{Im}\left[\begin{array}{cccc}
1 & -1 & 1 & -1 \\
0 & 0 & 1 & -1 \\
0 & 1 & -1 & 0 \\
0 & 0 & 0 & 0
\end{array}\right]
$$

and

$$
\mathcal{X}_{a}=\operatorname{Im}\left[G_{a} \mathcal{C}_{d} \quad B_{a}\right]=\operatorname{Im}\left[\begin{array}{ccccc}
0 & 0 & 0 & 0 & 0 \\
0 & 0 & 0 & 0 & 0 \\
0 & 0 & 0 & 0 & 0 \\
-1 & 1 & -1 & 1 & 1
\end{array}\right],
$$

and it has dimension four.

\section{CONClusion}

In this work we have discussed how to compute the reachable set of a descriptor system. A derivative-array approach is used to obtain a strangeness-free behavior system associated with the original DS. The resulting transformed system is then decoupled into a differential part and an algebraic part with the help of a projection method. The coefficient matrices of the projected system are used to define two subspaces and finally we have shown that the addition of these two subspaces is the reachable space of the original DS from the origin. We have demonstrated the proposed approach by considering an electrical circuit as a example.

\section{REFERENCES}

[1] B. Pal and B. Chaudhuri, Robust control in power systems. Springer : New York, Power electronics and power systems, 2005.

[2] K. E. Brenan, S. L. Campbell, and L. R. Petzold, Numerical Solution of Initial-Value Problems in Differential Algebraic Equations. Philadelphia, PA: SIAM Publications, 2nd ed., 1996.

[3] W. Blajer and K. Kołodziejczyk, "Control of underactuated mechanical systems with servo-constraints," Nonlinear Dynamics, vol. 50, no. 4, pp. 781-791, 2007.

[4] S. L. Campbell, P. Kunkel, and V. Mehrmann, Control and Optimization with Differential-Algebraic Constraints, ch. Regularization of Linear and Nonlinear Descriptor Systems. SIAM, 2012.

[5] L. Dai, Singular Control Systems. Berlin, Germany: Springer-Verlag, 1989.

[6] P. Kunkel and V. Mehrmann, Differential-Algebraic Equations. Analysis and Numerical Solution. Zürich, Switzerland: EMS Publishing House, 2006.

[7] V. Mehrmann, "Index concepts for differential-algebraic equations," Preprint 03-2012, Institut für Mathematik, TU Berlin, Str. des 17. Juni 136, D-10623 Berlin, FRG, 2012.

[8] P. Sauer and M. Pai, Power System Dynamics and Stability. Prentice Hall, Upper Saddle River, NJ, 1998.

[9] G. R. Duan, Analysis and Design of Descriptor Linear Systems. New York, NY: Springer-Verlag, 2010.

[10] A. Bunse-Gerstner, R. Byers, V. Mehrmann, and N. K. Nichols, "Numerical computation of an analytic singular value decomposition of a matrix valued function," Numer. Math., vol. 60, pp. 1-40, 1991.

[11] T. Stykel, Analysis and Numerical Solution of Generalized Lyapunov Equations. Dissertation, Institut für Mathematik, TU Berlin, Berlin, Germany, 2002.

[12] T. Berger, On differential-algebraic control systems. PhD thesis, Technische Universität Ilmennau, 2014. Dissertation.

[13] J. W. Demmel and B. Kågström, "Computing stable eigendecompositions of matrix pencils," Lin. Alg. Appl., vol. 88, pp. 139-186, 1987.

[14] J. W. Polderman and J. C. Willems, Introduction to Mathematical Systems Theory: A Behavioural Approach. New York, NY: SpringerVerlag, 1998.

[15] A. K. Baum and V. Mehrmann, "Positivity characterization of nonlinear DAEs, part II : decomposition of DAEs using projections," preprint, Institut für Mathematik, TU Berlin, Berlin, Germany, 2013.

[16] R. Lamour, R. Marz, and C. Tischendorf, Differential-Algebraic Equations: A Projector Based Analysis. Springer-Verlag, Berlin, 2013.

[17] G. H. Golub and C. F. Van Loan, Matrix Computations. Baltimore, MD: The Johns Hopkins University Press, 3rd ed., 1996. 